\title{
ON THE $M$-MATRIX INVERSE PROBLEM FOR SINGULAR AND SYMMETRIC JACOBI MATRICES *
}

\author{
ÁNGELES CARMONA ${ }^{\dagger}$, ANDRÉS M. ENCINAS ${ }^{\dagger}$, AND MARGARIDA MITJANA $\ddagger$
}

\begin{abstract}
A well-known property of an irreducible singular $M$-matrix is that it has a generalized inverse which is non-negative, but this is not always true for any generalized inverse. The authors have characterized when the Moore-Penrose inverse of a symmetric, singular, irreducible and tridiagonal $M$-matrix is itself an $M$-matrix. We aim here at giving new explicit examples of infinite families of matrices with order up to 4 having this property.
\end{abstract}

Key words. $M$-matrix, Moore-Penrose inverse, Schrödinger operator, Jacobi matrix.

AMS subject classifications. 05C50, 15A09

1. Introduction. Symmetric and irreducible Jacobi $M$-matrices are positive semi-definite matrices that can be expressed as $\mathrm{J}=\mathrm{D}-\mathrm{A}$, where $\mathrm{D}>0$ is a diagonal matrix and $\mathrm{A} \geq 0$ is a symmetric, tridiagonal and irreducible matrix. They appear in relation with self-adjoint boundary value problems for second order linear difference equations. In addition, the relation between Jacobi matrices and real orthogonal polynomials is widely known. On the other hand, symmetric and irreducible Jacobi $M$-matrices are identified with positive semi-definite Schrödinger operators on weighted paths, see for instance [2, 3]. They are also identified with perturbed Laplacians, see [1].

It is well-known that any irreducible singular $M$-matrix has a generalized inverse which is non-negative, but this is not always true for any generalized inverse, see [2, 5. Actually, the upper-right entry of the Moore-Penrose inverse of any symmetric Jacobi $M$-matrix is negative. The authors characterized in [2] when the Moore-Penrose inverse of a symmetric and irreducible Jacobi $M$-matrix has all its off-diagonal entries non-positive, which in turn is equivalent to the fact that the Moore-Penrose inverse is also an $M$-matrix. In addition, a deeper study of this problem was carried out

${ }^{*}$ Received by the editors on December 27, 2011. Accepted for publication on January 21, 2013 Handling Editor: Shmuel Friedland.

${ }^{\dagger}$ Departament de Matemàtica Aplicada III, Universitat Politècnica de Catalunya, 08034 Barcelona, Spain (angeles.carmona@upc.edu), (andres.marcos.encinas@upc.edu). Supported by the Spanish Research Council (Comisión Interministerial de Ciencia y Tecnología) under project MTM2010-19660

${ }^{\ddagger}$ Departament de Matemàtica Aplicada I, Universitat Politècnica de Catalunya, 08034 Barcelona, Spain (margarida.mitjana@upc.edu). Supported by the Spanish Research Council (Comisión Interministerial de Ciencia y Tecnología) under project MTM2011-28800-C02-01. 
in [3], where we showed that there exist infinite singular, symmetric and irreducible Jacobi $M$-matrices of any order whose Moore-Penrose inverse is also an $M$-matrix. This represented a wide improvement of the results obtained in [4. However, closed formulas for this class of matrices was obtained only for $n=2,3$. For instance, from [3. Corollary 2.4], the set of symmetric, singular and irreducible Jacobi $M$-matrices of order 3 such that their Moore-Penrose inverse are also an $M$-matrix is described by

$$
J=\left[\begin{array}{ccc}
\frac{c_{1} \sqrt{1-\left(1+t^{2}\right) x^{2}}}{x} & -c_{1} & 0 \\
-c_{1} & \frac{x\left(t c_{2}+c_{1}\right)}{\sqrt{1-\left(1+t^{2}\right) x^{2}}} & -c_{2} \\
0 & -c_{2} & \frac{c_{2} \sqrt{1-\left(1+t^{2}\right) x^{2}}}{t x}
\end{array}\right]
$$

where $c_{1}, c_{2}, t>0$ and $0<x \leq \sqrt{\frac{\min \left\{t c_{1}, c_{2}\right\}}{c_{2}+t^{3} c_{1}}}$, where the last inequality must be an strict inequality for $t=\frac{c_{2}}{c_{1}}$. In addition,

$$
J^{\dagger}=\frac{x}{c_{1} c_{2}}\left[\begin{array}{ccc}
\frac{c_{2}\left(1-x^{2}\right)^{2}+t^{3} x^{4} c_{1}}{\sqrt{1-\left(1+t^{2}\right) x^{2}}} & -x\left[c_{2}-x^{2}\left(c_{2}+t^{3} c_{1}\right)\right] & -\frac{t x^{2}\left[c_{2}+t c_{1}-x^{2}\left(c_{2}+t^{3} c_{1}\right)\right]}{\sqrt{1-\left(1+t^{2}\right) x^{2}}} \\
-x\left[c_{2}-x^{2}\left(c_{2}+t^{3} c_{1}\right)\right] & x^{2}\left(c_{2}+t^{3} c_{1}\right) \sqrt{1-\left(1+t^{2}\right) x^{2}} & -t x\left[c_{1} t-x^{2}\left(c_{2}+t^{3} c_{1}\right)\right] \\
-\frac{t x^{2}\left[c_{2}+t c_{1}-x^{2}\left(c_{2}+t^{3} c_{1}\right)\right]}{\sqrt{1-\left(1+t^{2}\right) x^{2}}} & -t x\left[c_{1} t-x^{2}\left(c_{2}+t^{3} c_{1}\right)\right] & \frac{t\left[c_{2} t x^{4}+c_{1}\left(1-t^{2} x^{2}\right)^{2}\right]}{\sqrt{1-\left(1+t^{2}\right) x^{2}}}
\end{array}\right] .
$$

Observe that either $c_{2}-x^{2}\left(c_{2}+t^{3} c_{1}\right)>0$ or $t c_{1}-x^{2}\left(c_{2}+t^{3} c_{1}\right)>0$, so $g_{12}<0$ or $g_{23}<0$, where $\mathrm{J}^{\dagger}=\left(g_{i j}\right)$.

In particular, $\mathrm{J}$ is the combinatorial Laplacian for the weighted path on three vertices whose conductances are given by $c_{1}$ and $c_{2}$ iff $t=1$ and $x=\sqrt{\frac{1}{3}}$ and hence the property of $\mathrm{J}^{\dagger}$ being an $M$-matrix holds iff $\frac{1}{2} \leq \frac{c_{2}}{c_{1}} \leq 2$.

The goal of this work is to carry out a deep study of the case $n=4$, providing explicit examples of a wide range of families of Jacobi $M$-matrices whose MoorePenrose inverse is also an $M$-matrix.

2. The $M$-inverse problem for $n=4$. The authors proved in 2 that any symmetric, irreducible and singular Jacobi $M$-matrix is given by

$$
\mathrm{J}(\mathrm{c}, \omega)=\left[\begin{array}{cccc}
\frac{c_{1} \omega_{2}}{\omega_{1}} & -c_{1} & 0 & 0 \\
-c_{1} & \frac{c_{2} \omega_{3}+c_{1} \omega_{1}}{\omega_{2}} & -c_{2} & 0 \\
0 & -c_{2} & \frac{c_{3} \omega_{4}+c_{2} \omega_{2}}{\omega_{3}} & -c_{3} \\
0 & 0 & -c_{3} & \frac{c_{3} \omega_{3}}{\omega_{4}}
\end{array}\right]
$$


where $\mathrm{c}=\left(c_{1}, c_{2}, c_{3}\right) \in(0,+\infty)^{3}$ and $\omega=\left(\omega_{1}, \omega_{2}, \omega_{3}, \omega_{4}\right) \in(0,+\infty)^{4}$ such that $\omega_{1}^{2}+\omega_{2}^{2}+\omega_{3}^{2}+\omega_{4}^{2}=1$ are called conductance and weight, respectively. Let

$$
\Omega=\left\{\omega=\left(\omega_{1}, \omega_{2}, \omega_{3}, \omega_{4}\right) \in(0,+\infty)^{4}: \omega_{1}^{2}+\omega_{2}^{2}+\omega_{3}^{2}+\omega_{4}^{2}=1\right\}
$$

be the set of weights. We recall that $\mathrm{J}(\mathrm{c}, \omega)$ can be seen as the matrix associated with a singular and positive semi-definite Schrödinger operator on the weighted path on four vertices with conductances $c_{1}, c_{2}, c_{3}$, see [2, 3] as Figure 2.1] shows.

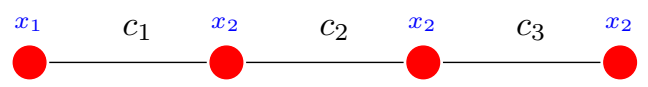

FIG. 2.1. Weighted path on four vertices

In particular, $\mathrm{J}(\mathrm{c}, \omega)$ coincides with the combinatorial Laplacian of the weighted path iff the weight is constant; that is, when $\omega_{i}=\frac{1}{2}$, for any $i=1,2,3,4$. Moreover, in [2, Corollary 5.2], we obtained that $\mathrm{J}^{\dagger}(\mathrm{c}, \omega)=\left(g_{i j}\right)$, where $g_{i j}=g_{j i}$ and

$$
\begin{aligned}
& g_{11}=\omega_{1}^{2}\left[\frac{\left(\omega_{2}^{2}+\omega_{3}^{2}+\omega_{4}^{2}\right)^{2}}{c_{1} \omega_{1} \omega_{2}}+\frac{\left(\omega_{3}^{2}+\omega_{4}^{2}\right)^{2}}{c_{2} \omega_{2} \omega_{3}}+\frac{\omega_{4}^{4}}{c_{3} \omega_{3} \omega_{4}}\right] \\
& g_{12}=\omega_{1} \omega_{2}\left[\frac{\left(\omega_{3}^{2}+\omega_{4}^{2}\right)^{2}}{c_{2} \omega_{2} \omega_{3}}+\frac{\omega_{4}^{4}}{c_{3} \omega_{3} \omega_{4}}-\frac{\omega_{1}^{2}\left(\omega_{2}^{2}+\omega_{3}^{2}+\omega_{4}^{2}\right)}{c_{1} \omega_{1} \omega_{2}}\right] \\
& g_{13}=\omega_{1} \omega_{3}\left[\frac{\omega_{4}^{4}}{c_{3} \omega_{3} \omega_{4}}-\frac{\omega_{1}^{2}\left(\omega_{2}^{2}+\omega_{3}^{2}+\omega_{4}^{2}\right)}{c_{1} \omega_{1} \omega_{2}}-\frac{\left(\omega_{1}^{2}+\omega_{2}^{2}\right)\left(\omega_{3}^{2}+\omega_{4}^{2}\right)}{c_{2} \omega_{2} \omega_{3}}\right] \\
& g_{14}=-\omega_{1} \omega_{4}\left[\frac{\omega_{1}^{2}\left(\omega_{2}^{2}+\omega_{3}^{2}+\omega_{4}^{2}\right)}{c_{1} \omega_{1} \omega_{2}}+\frac{\left(\omega_{1}^{2}+\omega_{2}^{2}\right)\left(\omega_{3}^{2}+\omega_{4}^{2}\right)}{c_{2} \omega_{2} \omega_{3}}+\frac{\left(\omega_{1}^{2}+\omega_{2}^{2}+\omega_{3}^{2}\right) \omega_{4}^{2}}{c_{3} \omega_{3} \omega_{4}}\right] \\
& g_{22}=\omega_{2}^{2}\left[\frac{\omega_{1}^{4}}{c_{1} \omega_{1} \omega_{2}}+\frac{\left(\omega_{3}^{2}+\omega_{4}^{2}\right)^{2}}{c_{2} \omega_{2} \omega_{3}}+\frac{\omega_{4}^{4}}{c_{3} \omega_{3} \omega_{4}}\right] \\
& g_{23}=\omega_{2} \omega_{3}\left[\frac{\omega_{1}^{4}}{c_{1} \omega_{1} \omega_{2}}+\frac{\omega_{4}^{4}}{c_{3} \omega_{3} \omega_{4}}-\frac{\left(\omega_{1}^{2}+\omega_{2}^{2}\right)\left(\omega_{3}^{2}+\omega_{4}^{2}\right)}{c_{2} \omega_{2} \omega_{3}}\right] \\
& g_{24}=\omega_{2} \omega_{4}\left[\frac{\omega_{1}^{4}}{c_{1} \omega_{1} \omega_{2}}-\frac{\left(\omega_{1}^{2}+\omega_{2}^{2}\right)\left(\omega_{3}^{2}+\omega_{4}^{2}\right)}{c_{2} \omega_{2} \omega_{3}}-\frac{\left(\omega_{1}^{2}+\omega_{2}^{2}+\omega_{3}^{2}\right) \omega_{4}^{2}}{c_{3} \omega_{3} \omega_{4}}\right] \\
& g_{33}=\omega_{3}^{2}\left[\frac{\omega_{1}^{4}}{c_{1} \omega_{1} \omega_{2}}+\frac{\left(\omega_{1}^{2}+\omega_{2}^{2}\right)^{2}}{c_{2} \omega_{2} \omega_{3}}+\frac{\omega_{4}^{4}}{c_{3} \omega_{3} \omega_{4}}\right] \\
& g_{34}=\omega_{3} \omega_{4}\left[\frac{\omega_{1}^{4}}{c_{1} \omega_{1} \omega_{2}}+\frac{\left(\omega_{1}^{2}+\omega_{2}^{2}\right)^{2}}{c_{2} \omega_{2} \omega_{3}}-\frac{\left(\omega_{1}^{2}+\omega_{2}^{2}+\omega_{3}^{2}\right) \omega_{4}^{2}}{c_{3} \omega_{3} \omega_{4}}\right] \\
& g_{44}=\omega_{4}^{2}\left[\frac{\omega_{1}^{4}}{c_{1} \omega_{1} \omega_{2}}+\frac{\left(\omega_{1}^{2}+\omega_{2}^{2}\right)^{2}}{c_{2} \omega_{2} \omega_{3}}+\frac{\left(\omega_{1}^{2}+\omega_{2}^{2}+\omega_{3}^{2}\right)^{2}}{c_{3} \omega_{3} \omega_{4}}\right] .
\end{aligned}
$$

A conductance $\mathrm{c}$, is called feasible if there exists a weight $\omega$ such that $\mathrm{J}^{\dagger}(\mathrm{c}, \omega)$ is an $M$-matrix. From Identities (2.2), we obtain that $\mathrm{J}^{\dagger}(\mathrm{c}, \omega)$ is an $M$-matrix iff 
$g_{12}, g_{23}, g_{34} \leq 0$; that is, iff there exist $x, y, z \in \mathbb{R}$ such that

$$
\begin{aligned}
& \frac{\omega_{1}^{2}\left(\omega_{2}^{2}+\omega_{3}^{2}+\omega_{4}^{2}\right)}{c_{1} \omega_{1} \omega_{2}}-\frac{\left(\omega_{3}^{2}+\omega_{4}^{2}\right)^{2}}{c_{2} \omega_{2} \omega_{3}}-\frac{\omega_{4}^{4}}{c_{3} \omega_{3} \omega_{4}}=x^{2} \\
&-\frac{\omega_{1}^{4}}{c_{1} \omega_{1} \omega_{2}}+\frac{\left(\omega_{1}^{2}+\omega_{2}^{2}\right)\left(\omega_{3}^{2}+\omega_{4}^{2}\right)}{c_{2} \omega_{2} \omega_{3}}-\frac{\omega_{4}^{4}}{c_{3} \omega_{3} \omega_{4}}=y^{2} \\
&-\frac{\omega_{1}^{4}}{c_{1} \omega_{1} \omega_{2}}-\frac{\left(\omega_{1}^{2}+\omega_{2}^{2}\right)^{2}}{c_{2} \omega_{2} \omega_{3}}+\frac{\left(\omega_{1}^{2}+\omega_{2}^{2}+\omega_{3}^{2}\right) \omega_{4}^{2}}{c_{3} \omega_{3} \omega_{4}}=z^{2}
\end{aligned}
$$

which is equivalent to the system

$$
\begin{aligned}
& \frac{\omega_{1}}{c_{1} \omega_{2}}-\frac{\left(\omega_{3}^{2}+\omega_{4}^{2}\right)}{c_{2} \omega_{2} \omega_{3}}=x^{2}-y^{2} \\
& \frac{\left(\omega_{1}^{2}+\omega_{2}^{2}\right)}{c_{2} \omega_{2} \omega_{3}}-\frac{\omega_{4}}{c_{3} \omega_{3}}=y^{2}-z^{2} \\
& \frac{\omega_{4}\left(\omega_{2}^{2} \omega_{3}^{2}-\omega_{1}^{2} \omega_{4}^{2}\right)}{c_{3} \omega_{3}}=\left(\omega_{1}^{2}+\omega_{2}^{2}\right)\left(\omega_{1}^{2} x^{2}+\omega_{2}^{2} y^{2}\right)+\left(\omega_{3}^{2}+\omega_{4}^{2}\right)\left(\omega_{1}^{2} y^{2}+\omega_{2}^{2} z^{2}\right) .
\end{aligned}
$$

The non-negativity of the right side of the third equality implies that $\omega_{1} \omega_{4} \leq \omega_{2} \omega_{3}$ is a necessary condition for $\mathrm{J}^{\dagger}(\mathrm{c}, \omega)$ be an $M$-matrix. For this reason, in what follows we consider the set of weights

$$
\Omega_{0}=\left\{\omega \in \Omega: \omega_{1} \omega_{4}=\omega_{2} \omega_{3}\right\} \quad \text { and } \quad \Omega_{+}=\left\{\omega \in \Omega: \omega_{1} \omega_{4}<\omega_{2} \omega_{3}\right\} .
$$

When $\omega \in \Omega_{0}$ then, $x=y=z=0$. It turns out that $g_{12}=g_{23}=g_{34}=0$ and that $\mathrm{J}^{\dagger}(\mathrm{c}, \omega)$ is an $M$-matrix iff the conductance and the weight are related by the following identities

$$
c_{1}=\frac{c_{2} \omega_{1} \omega_{3}}{\omega_{3}^{2}+\omega_{4}^{2}} \quad \text { and } \quad c_{3}=\frac{c_{2} \omega_{2} \omega_{4}}{\omega_{1}^{2}+\omega_{2}^{2}}
$$

When $\omega \in \Omega_{+}$, then changing $x, y, z$ by $x \sqrt{\frac{\omega_{2}^{2} \omega_{3}^{2}-\omega_{1}^{2} \omega_{4}^{2}}{\omega_{2} \omega_{3}}}, y \sqrt{\frac{\omega_{2}^{2} \omega_{3}^{2}-\omega_{1}^{2} \omega_{4}^{2}}{\omega_{2} \omega_{3}}}$ and $z \sqrt{\frac{\omega_{2}^{2} \omega_{3}^{2}-\omega_{1}^{2} \omega_{4}^{2}}{\omega_{2} \omega_{3}}}$, respectively, we obtain that System (2.3) implies that $\mathrm{J}^{\dagger}(\mathrm{c}, \omega)$ is an $M$-matrix iff the conductance and the weight are related by the identities

$$
\begin{aligned}
& c_{1}=\frac{\omega_{1} \omega_{3}}{\omega_{3}^{2}\left(\omega_{1}^{2}+\omega_{2}^{2}\right) x^{2}+\left[\omega_{4}^{2}\left(\omega_{1}^{2}+\omega_{2}^{2}\right)+\omega_{3}^{2}\left(\omega_{3}^{2}+\omega_{4}^{2}\right)\right] y^{2}+\omega_{4}^{2}\left(\omega_{3}^{2}+\omega_{4}^{2}\right) z^{2}}, \\
& c_{2}=\frac{1}{\omega_{1}^{2} x^{2}+\left(\omega_{2}^{2}+\omega_{3}^{2}\right) y^{2}+\omega_{4}^{2} z^{2}}, \\
& c_{3}=\frac{\omega_{2} \omega_{4}}{\omega_{1}^{2}\left(\omega_{1}^{2}+\omega_{2}^{2}\right) x^{2}+\left[\omega_{1}^{2}\left(\omega_{3}^{2}+\omega_{4}^{2}\right)+\omega_{2}^{2}\left(\omega_{1}^{2}+\omega_{2}^{2}\right)\right] y^{2}+\omega_{2}^{2}\left(\omega_{3}^{2}+\omega_{4}^{2}\right) z^{2}},
\end{aligned}
$$


where $x, y, z \in \mathbb{R}$ are such that $x^{2}+y^{2}+z^{2}>0$. These identities, together with (2.4), determine all the feasible conductances for the raised problem. In particular, we study the cases corresponding to the choices $x=y=z, y=z=0, x=y=0$ and finally $x=z=0$.

The choice $x=y=z$ leads to

$$
x^{2}=\frac{1}{c_{2}}, \quad c_{1}=\frac{c_{2} \omega_{1} \omega_{3}}{\omega_{3}^{2}+\omega_{4}^{2}} \quad \text { and } \quad c_{3}=\frac{c_{2} \omega_{2} \omega_{4}}{\omega_{1}^{2}+\omega_{2}^{2}}
$$

the same expressions in (2.4), for weights in $\Omega_{0}$. This fact allows us to consider both cases simultaneously. Therefore, for any $\omega \in \Omega_{0} \cup \Omega_{+}$we define the conductance

$$
\mathrm{c}(\omega)=\left(\frac{\omega_{1} \omega_{3}}{\omega_{3}^{2}+\omega_{4}^{2}}, 1, \frac{\omega_{2} \omega_{4}}{\omega_{1}^{2}+\omega_{2}^{2}}\right) .
$$

When $y=z=0$, System (2.5) becomes

$$
x^{2}=\frac{1}{c_{2} \omega_{1}^{2}}, \quad c_{1}=\frac{\omega_{1}^{3} c_{2}}{\omega_{3}\left(\omega_{1}^{2}+\omega_{2}^{2}\right)} \quad \text { and } \quad c_{3}=\frac{\omega_{2} \omega_{4} c_{2}}{\omega_{1}^{2}+\omega_{2}^{2}}
$$

and hence, for any $\omega \in \Omega_{+}$we define the conductance

$$
c_{x}(\omega)=\left(\frac{\omega_{1}^{3}}{\omega_{3}\left(\omega_{1}^{2}+\omega_{2}^{2}\right)}, 1, \frac{\omega_{2} \omega_{4}}{\omega_{1}^{2}+\omega_{2}^{2}}\right) .
$$

When $x=y=0$, System (2.5) reads as

$$
z^{2}=\frac{1}{c_{2} \omega_{4}^{2}}, \quad c_{1}=\frac{\omega_{1} \omega_{3} c_{2}}{\omega_{3}^{2}+\omega_{4}^{2}} \quad \text { and } \quad c_{3}=\frac{\omega_{4}^{3} c_{2}}{\omega_{2}\left(\omega_{3}^{2}+\omega_{4}^{2}\right)}
$$

and hence, for any $\omega \in \Omega_{+}$we define the conductance

$$
\mathrm{c}_{z}(\omega)=\left(\frac{\omega_{1} \omega_{3}}{\omega_{3}^{2}+\omega_{4}^{2}}, 1, \frac{\omega_{4}^{3}}{\omega_{2}\left(\omega_{3}^{2}+\omega_{4}^{2}\right)}\right) .
$$

When $x=z=0$, System (2.5) determines that $y^{2}=\frac{1}{c_{2}\left(\omega_{2}^{2}+\omega_{3}^{2}\right)}$,

$$
c_{1}=\frac{c_{2} \omega_{1} \omega_{3}\left(\omega_{2}^{2}+\omega_{3}^{2}\right)}{\omega_{4}^{2}\left(\omega_{1}^{2}+\omega_{2}^{2}\right)+\omega_{3}^{2}\left(\omega_{3}^{2}+\omega_{4}^{2}\right)} \quad \text { and } \quad c_{3}=\frac{c_{2} \omega_{2} \omega_{4}\left(\omega_{2}^{2}+\omega_{3}^{2}\right)}{\omega_{1}^{2}\left(\omega_{3}^{2}+\omega_{4}^{2}\right)+\omega_{2}^{2}\left(\omega_{1}^{2}+\omega_{2}^{2}\right)}
$$

and hence, for any $\omega \in \Omega_{+}$we define the conductance

$$
c_{y}(\omega)=\left(\frac{\omega_{1} \omega_{3}\left(\omega_{2}^{2}+\omega_{3}^{2}\right)}{\omega_{4}^{2}\left(\omega_{1}^{2}+\omega_{2}^{2}\right)+\omega_{3}^{2}\left(\omega_{3}^{2}+\omega_{4}^{2}\right)}, 1, \frac{\omega_{2} \omega_{4}\left(\omega_{2}^{2}+\omega_{3}^{2}\right)}{\omega_{1}^{2}\left(\omega_{3}^{2}+\omega_{4}^{2}\right)+\omega_{2}^{2}\left(\omega_{1}^{2}+\omega_{2}^{2}\right)}\right) .
$$


Next, we summarize the above results.

Proposition 2.1. Given a conductance c, if $\mathrm{J}^{\dagger}(\mathrm{c}, \omega)$ is an $M$-matrix then $\omega \in$ $\Omega_{0} \cup \Omega_{+}$. Conversely, given $\omega \in \Omega_{0} \cup \Omega_{+}$, then $\mathrm{J}^{\dagger}(\mathrm{tc}(\omega), \omega)$, where $t>0$, is an $M$-matrix and when $\omega \in \Omega_{0}$ the only conductances with this property are $t \mathrm{c}(\omega)$ where $t>0$. In addition, given $\omega \in \Omega_{+}$, then for any $t>0, \mathrm{~J}^{\dagger}\left(t \mathrm{c}_{x}(\omega), \omega\right), \mathrm{J}^{\dagger}\left(t \mathrm{c}_{y}(\omega), \omega\right)$ and $\mathrm{J}^{\dagger}\left(t \mathrm{c}_{z}(\omega), \omega\right)$ are $M$-matrices.

3. Jacobi matrices of the form $\mathrm{J}(t \mathrm{c}(\omega), \omega), \mathrm{J}\left(t \mathrm{c}_{x}(\omega), \omega\right)$ and $\mathrm{J}\left(t \mathrm{c}_{z}(\omega), \omega\right)$. Our next aim is to characterize the feasible conductances of the form $c=t c(\omega)$ where $t>0$ and $\omega \in \Omega_{0}$ and the conductances of the form $\mathrm{c}=t \mathrm{c}(\omega), \mathrm{c}=t \mathrm{c}_{x}(\omega)$ or $\mathrm{c}=t \mathrm{c}_{z}(\omega)$ where $t>0$ and $\omega \in \Omega_{+}$.

First, if $\mathrm{c}=\left(c_{1}, c_{2}, c_{3}\right)$ and $\mathrm{c}=c_{2} \mathrm{c}(\omega)$ for some $\omega \in \Omega_{0} \cup \Omega_{+}$, the well-known Young's Inequality $a^{2}+b^{2} \geq 2|a b|$ implies that $c_{2}^{2} \geq 4 c_{1} c_{3}$ and the equality holds iff $\omega_{1}=\omega_{2}$ and $\omega_{3}=\omega_{4}$; which in particular implies that $\omega \in \Omega_{0}$. So, we obtain the following result.

THEOREM 3.1. If $\mathrm{c}=\left(c_{1}, c_{2}, c_{3}\right)$ and $c_{2}=2 \sqrt{c_{1} c_{3}}$, then

$$
\mathrm{J}_{\mathrm{c}}=\left[\begin{array}{cccc}
c_{1} & -c_{1} & 0 & 0 \\
-c_{1} & c_{1}+2 c_{3} & -2 \sqrt{c_{1} c_{3}} & 0 \\
0 & -2 \sqrt{c_{1} c_{3}} & 2 c_{1}+c_{3} & -c_{3} \\
0 & 0 & -c_{3} & c_{3}
\end{array}\right]
$$

is the only Jacobi matrix of the form $\mathrm{J}(\mathrm{c}, \omega)$ where $\omega \in \Omega_{0}$, such that its MoorePenrose inverse is an $M-$ matrix. Moreover,

$$
\mathrm{J}_{\mathrm{c}}^{\dagger}=\left[\begin{array}{cccc}
\frac{c_{1}+2 c_{3}}{2 c_{1}\left(c_{1}+c_{3}\right)} & 0 & -\frac{\sqrt{c_{3}}}{2\left(c_{1}+c_{3}\right) \sqrt{c_{1}}} & -\frac{1}{2 \sqrt{c_{1} c_{3}}} \\
0 & \frac{1}{2\left(c_{1}+c_{3}\right)} & 0 & -\frac{\sqrt{c_{1}}}{2\left(c_{1}+c_{3}\right) \sqrt{c_{3}}} \\
-\frac{\sqrt{c_{3}}}{2\left(c_{1}+c_{3}\right) \sqrt{c_{1}}} & 0 & \frac{1}{2\left(c_{1}+c_{3}\right)} & 0 \\
-\frac{1}{2 \sqrt{c_{1} c_{3}}} & -\frac{\sqrt{c_{1}}}{2\left(c_{1}+c_{3}\right) \sqrt{c_{3}}} & 0 & \frac{2 c_{1}+c_{3}}{2 c_{3}\left(c_{1}+c_{3}\right)}
\end{array}\right]
$$

Proof. Proposition 2.1 implies that $\mathrm{c}=c_{2} \mathrm{c}(\omega)$. Moreover, the above reasoning concludes that necessarily $\omega_{1}=\omega_{2}$ and $\omega_{3}=\omega_{4}$, and then, we have $c_{1}=\frac{\sqrt{c_{1} c_{3}} \omega_{1}}{\omega_{3}}$ and $c_{3}=\frac{\sqrt{c_{1} c_{3}} \omega_{3}}{\omega_{1}}$. Therefore, $\omega_{3}=\omega_{1} \sqrt{\frac{c_{3}}{c_{1}}}$ and $1=\frac{2 \omega_{1}^{2}\left(c_{1}+c_{3}\right)}{c_{1}}$ so that, $\omega_{1}=$ $\sqrt{\frac{c_{1}}{2\left(c_{1}+c_{3}\right)}}$ and $\omega_{3}=\sqrt{\frac{c_{3}}{2\left(c_{1}+c_{3}\right)}}$. The result follows taking into account (2.1) and 
(2.2).

REMARK 3.2. If we consider $c_{1}=c_{3}=c$ in the above theorem, then $\mathrm{J}$ is nothing else but the combinatorial Laplacian of the weighted path whose conductance is $(c, 2 c, c)$.

If either $\mathrm{c}=c_{2} \mathrm{c}_{x}(\omega)$ or $\mathrm{c}=c_{2} \mathrm{c}_{z}(\omega)$ for some $\omega \in \Omega_{+}$, Young's Inequality implies that $4 c_{1} c_{3}<c_{2}^{2}$. Therefore, it will be useful to consider the 3 -dimensional cone, see Figure 3.1

$$
K=\left\{\left(c_{1}, c_{2}, c_{3}\right) \in(0,+\infty)^{3}: c_{2}^{2}>4 c_{1} c_{3}\right\},
$$

since $\mathrm{c}(\omega), \mathrm{c}_{x}(\omega), \mathrm{c}_{z}(\omega) \in K$ for any $\omega \in \Omega_{+}$and moreover $\mathrm{c}(\omega) \in K$ when $\omega \in \Omega_{0}$ but either $\omega_{1} \neq \omega_{2}$ or $\omega_{3} \neq \omega_{4}$. On the other hand, if $\omega_{1}=\omega_{2}$ and $\omega_{3}=\omega_{4}$, then $\mathrm{c}(\omega) \in \partial K$.

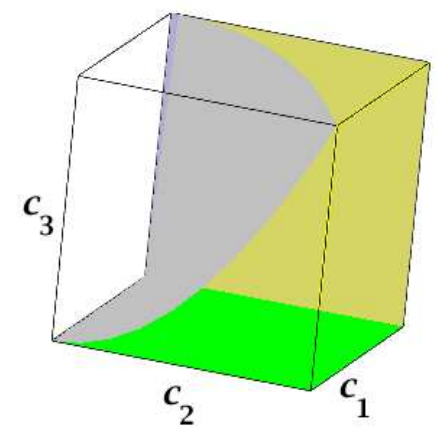

Fig. 3.1. Cone of Feasible Conductances

Given $\mathrm{c}=\left(c_{1}, c_{2}, c_{3}\right) \in K$, we consider the values $\tau_{\mathrm{c}}^{1}=\frac{2 c_{3}}{c_{2}}, \tau_{\mathrm{c}}^{2}=\frac{c_{2}}{2 c_{1}}$ and $\xi_{\mathrm{c}}=\sqrt{\frac{c_{3}}{c_{1}}}$ that clearly satisfy $0<\tau_{\mathrm{c}}^{1}<\xi_{\mathrm{c}}<\tau_{\mathrm{c}}^{2}$. Moreover, we also consider the functions $a_{\mathrm{c}}^{ \pm}:\left(0, \tau_{\mathrm{c}}^{2}\right] \longrightarrow(0,+\infty)$ and $b_{\mathrm{c}}^{ \pm}:\left[\tau_{\mathrm{c}}^{1},+\infty\right) \longrightarrow(0,+\infty)$ defined respectively for any $s \in\left(0, \tau_{\mathrm{c}}^{2}\right]$ or any $s \in\left[\tau_{\mathrm{c}}^{1},+\infty\right)$ as

$$
a_{\mathrm{c}}^{ \pm}(s)=\frac{1}{2 c_{1}}\left[c_{2} \pm \sqrt{c_{2}^{2}-4 c_{1}^{2} s^{2}}\right] \quad \text { and } \quad b_{\mathrm{c}}^{ \pm}(s)=\frac{1}{2 c_{3}}\left[c_{2} s \pm \sqrt{c_{2}^{2} s^{2}-4 c_{3}^{2}}\right] .
$$

Clearly the common domain of the above functions is $\left[\tau_{\mathrm{c}}^{1}, \tau_{\mathrm{c}}^{2}\right]$. The main properties of functions $a_{\mathrm{c}}^{ \pm}$and $b_{\mathrm{c}}^{ \pm}$are described in the statements of the following lemmas, and 
all of them have a straightforward proof.

LEMMA 3.3. Given $\mathrm{c} \in K$, the following properties hold:

(i) $a_{\mathrm{c}}^{ \pm}\left(\tau_{\mathrm{c}}^{1}\right)=\frac{c_{2}^{2} \pm \sqrt{c_{2}^{4}-16 c_{1}^{2} c_{3}^{2}}}{2 c_{1} c_{2}}, a_{\mathrm{c}}^{ \pm}\left(\xi_{\mathrm{c}}\right)=\frac{c_{2} \pm \sqrt{c_{2}^{2}-4 c_{1} c_{3}}}{2 c_{1}}$ and $a_{\mathrm{c}}^{ \pm}\left(\tau_{\mathrm{c}}^{2}\right)=\tau_{\mathrm{c}}^{2}$.

(ii) $b_{\mathrm{c}}^{ \pm}\left(\tau_{\mathrm{c}}^{1}\right)=1, b_{\mathrm{c}}^{ \pm}\left(\xi_{\mathrm{c}}\right)=\frac{c_{2} \pm \sqrt{c_{2}^{2}-4 c_{1} c_{3}}}{2 \sqrt{c_{1} c_{3}}}$ and $b_{\mathrm{c}}^{ \pm}\left(\tau_{\mathrm{c}}^{2}\right)=\frac{c_{2}^{2} \pm \sqrt{c_{2}^{4}-16 c_{1}^{2} c_{3}^{2}}}{4 c_{1} c_{3}}$.

(iii) $\frac{1}{a_{\mathrm{c}}^{ \pm}(s)}=\frac{a_{\mathrm{c}}^{\mp}(s)}{s^{2}}$ and $\left(a_{\mathrm{c}}^{ \pm}(s)\right)^{2}=\frac{c_{2} a_{\mathrm{c}}^{ \pm}(s)}{c_{1}}-s^{2}$ for any $s \in\left(0, \tau_{\mathrm{c}}^{2}\right]$.

(iv) $\frac{1}{b_{\mathrm{c}}^{ \pm}(s)}=b_{\mathrm{c}}^{\mp}(s)$ and $\left(b_{\mathrm{c}}^{ \pm}(s)\right)^{2}=\frac{c_{2} s b_{\mathrm{c}}^{ \pm}(s)}{c_{3}}-1$, for any $s \in\left[\tau_{\mathrm{c}}^{1},+\infty\right)$.

LEMMA 3.4. If $\mathrm{c} \in K$, the following properties hold:

(i) $\left(a_{\mathrm{c}}^{ \pm}\right)^{\prime}(s)=\frac{\mp 2 c_{1} s}{\sqrt{c_{2}^{2}-4 c_{1}^{2} s^{2}}}$ for any $s \in\left(0, \tau_{\mathrm{c}}^{2}\right)$, whereas $\left(b_{\mathrm{c}}^{ \pm}\right)^{\prime}(s)=\frac{ \pm c_{2} b_{\mathrm{c}}^{ \pm}(s)}{\sqrt{c_{2}^{2} s^{2}-4 c_{3}^{2}}}$ for any $s \in\left(\tau_{c}^{1},+\infty\right)$.

(ii) $a_{\mathrm{c}}^{+}(s) b_{\mathrm{c}}^{+}(s)>s$ for any $s \in\left[\tau_{\mathrm{c}}^{1}, \tau_{\mathrm{c}}^{2}\right]$.

(iii) $a_{\mathrm{c}}^{-}(s) b_{\mathrm{c}}^{-}(s)<s$ for any $s \in\left[\tau_{\mathrm{c}}^{1}, \tau_{\mathrm{c}}^{2}\right]$.

(iv) $a_{\mathrm{c}}^{+}(s) b_{\mathrm{c}}^{-}(s)>s$ for any $s \in\left[\tau_{\mathrm{c}}^{1}, \xi_{\mathrm{c}}\right)$ and $a_{\mathrm{c}}^{+}(s) b_{\mathrm{c}}^{-}(s) \leq s$ for any $s \in\left[\xi_{\mathrm{c}}, \tau_{\mathrm{c}}^{2}\right]$ with equality iff $s=\xi_{\mathrm{c}}$.

(iv) $a_{\mathrm{c}}^{-}(s) b_{\mathrm{c}}^{+}(s)<s$ for any $s \in\left[\tau_{\mathrm{c}}^{1}, \xi_{\mathrm{c}}\right)$ and $a_{\mathrm{c}}^{-}(s) b_{\mathrm{c}}^{+}(s) \geq s$ for any $s \in\left[\xi_{\mathrm{c}}, \tau_{\mathrm{c}}^{2}\right]$ with equality iff $s=\xi_{\mathrm{c}}$.

Now we are ready to establish the main result of this section.

THEOREM 3.5. If $\mathrm{c}=\left(c_{1}, c_{2}, c_{3}\right) \in K$ then, the following results hold:

$$
\mathrm{J}_{\mathrm{c}}^{1}(s)=\left[\begin{array}{cccc}
c_{1} b_{\mathrm{c}}^{+}(s) & -c_{1} & 0 & 0 \\
-c_{1} & b_{\mathrm{c}}^{-}(s)\left(c_{1}+c_{2} a_{\mathrm{c}}^{+}(s)\right) & -c_{2} & 0 \\
0 & -c_{2} & \frac{a_{\mathrm{c}}^{-}(s)\left(c_{3} s+c_{2} b_{\mathrm{c}}^{+}(s)\right)}{s^{2}} & -c_{3} \\
0 & 0 & -c_{3} & \frac{c_{3} a_{\mathrm{c}}^{+}(s)}{s}
\end{array}\right]
$$

for any $s \in\left[\tau_{\mathrm{c}}^{1}, \tau_{\mathrm{c}}^{2}\right]$,

$$
\mathrm{J}_{\mathrm{c}}^{2}(s)=\left[\begin{array}{cccc}
c_{1} b_{\mathrm{c}}^{-}(s) & -c_{1} & 0 & 0 \\
-c_{1} & b_{\mathrm{c}}^{+}(s)\left(c_{1}+c_{2} a_{\mathrm{c}}^{+}(s)\right) & -c_{2} & 0 \\
0 & -c_{2} & \frac{a_{\mathrm{c}}^{-}(s)\left(c_{3} s+c_{2} b_{\mathrm{c}}^{-}(s)\right)}{s^{2}} & -c_{3} \\
0 & 0 & -c_{3} & \frac{c_{3} a_{\mathrm{c}}^{+}(s)}{s}
\end{array}\right]
$$


for any $s \in\left[\tau_{\mathrm{c}}^{1}, \xi_{\mathrm{c}}\right]$ and

$$
\mathrm{J}_{\mathrm{c}}^{3}(s)=\left[\begin{array}{cccc}
c_{1} b_{\mathrm{c}}^{+}(s) & -c_{1} & 0 & 0 \\
-c_{1} & b_{\mathrm{c}}^{-}(s)\left(c_{1}+c_{2} a_{\mathrm{c}}^{-}(s)\right) & -c_{2} & 0 \\
0 & -c_{2} & \frac{a_{\mathrm{c}}^{+}(s)\left(c_{3} s+c_{2} b_{\mathrm{c}}^{+}(s)\right)}{s^{2}} & -c_{3} \\
0 & 0 & -c_{3} & \frac{c_{3} a_{\mathrm{c}}^{-}(s)}{s}
\end{array}\right]
$$

for any $s \in\left[\xi_{\mathrm{c}}, \tau_{\mathrm{c}}^{2}\right]$ are the only Jacobi matrices of the form $\mathrm{J}=\mathrm{J}\left(c_{2} \mathrm{c}(\omega), \omega\right)$ such that their Moore-Penrose inverses are $M$-matrices. Moreover, $\mathrm{J}_{\mathrm{c}}^{2}\left(\xi_{\mathrm{c}}\right)$ and $\mathrm{J}_{\mathrm{c}}^{3}\left(\xi_{\mathrm{c}}\right)$ are the only Jacobi matrices of the form $\mathrm{J}(\mathrm{c}, \omega)$ where $\omega \in \Omega_{0}$, such that their Moore-Penrose inverses are $M$-matrices. In addition,

$$
\mathrm{J}_{\mathrm{c}}^{4}(s)=\left[\begin{array}{cccc}
c_{1} b_{\mathrm{c}}^{+}(s) & -c_{1} & 0 & 0 \\
-c_{1} & \frac{s b_{\mathrm{c}}^{-}(s)\left(c_{1}^{2}+c_{2}^{2}\right)-c_{2} c_{3}}{c_{1} s} & -c_{2} & 0 \\
0 & -c_{2} & \frac{c_{1} s\left[s b_{\mathrm{c}}^{+}(s)\left(c_{2}^{2}+c_{3}^{2}\right)-c_{2} c_{3}\right]}{c_{3}^{2}} & -c_{3} \\
0 & 0 & -c_{3} & \frac{c_{3}^{2} b_{\mathrm{c}}^{-}(s)}{c_{1} s^{2}}
\end{array}\right]
$$

and

$$
\mathrm{J}_{\mathrm{c}}^{5}(s)=\left[\begin{array}{cccc}
c_{1} b_{\mathrm{c}}^{-}(s) & -c_{1} & 0 & 0 \\
-c_{1} & \frac{s b_{\mathrm{c}}^{+}(s)\left(c_{1}^{2}+c_{2}^{2}\right)-c_{2} c_{3}}{c_{1} s} & -c_{2} & 0 \\
0 & -c_{2} & \frac{c_{1} s\left[s b_{\mathrm{c}}^{-}(s)\left(c_{2}^{2}+c_{3}^{2}\right)-c_{2} c_{3}\right]}{c_{3}^{2}} & -c_{3} \\
0 & 0 & -c_{3} & \frac{c_{3}^{2} b_{\mathrm{c}}^{+}(s)}{c_{1} s^{2}}
\end{array}\right]
$$

for any $s \in\left[\tau_{\mathrm{c}}^{1}, \xi_{\mathrm{c}}\right)$ are the only Jacobi matrices of the form $\mathrm{J}=\mathrm{J}\left(c_{2} \mathrm{c}_{x}(\omega), \omega\right)$ such that their Moore-Penrose inverses are $M$-matrices. Finally,

$\mathrm{J}_{\mathrm{c}}^{6}(s)=\left[\begin{array}{cccc}\frac{c_{1}^{2} s a_{\mathrm{c}}^{-}(s)}{c_{3}} & -c_{1} & 0 & 0 \\ -c_{1} & \frac{c_{3}\left[a_{\mathrm{c}}^{+}(s)\left(c_{1}^{2}+c_{2}^{2}\right)-c_{1} c_{2} s^{2}\right]}{c_{1}^{2} s^{3}} & -c_{2} & 0 \\ 0 & -c_{2} & \frac{a_{c}^{-}(s)\left(c_{2}^{2}+c_{3}^{2}\right)-c_{1} c_{2} s^{2}}{c_{3} s} & -c_{3} \\ 0 & 0 & -c_{3} & \frac{c_{3} a_{c}^{+}(s)}{s}\end{array}\right]$ 
and

$$
J_{\mathrm{c}}^{7}(s)=\left[\begin{array}{cccc}
\frac{c_{1}^{2} s a_{\mathrm{c}}^{+}(s)}{c_{3}} & -c_{1} & 0 & 0 \\
-c_{1} & \frac{c_{3}\left[a_{\mathrm{c}}^{-}(s)\left(c_{1}^{2}+c_{2}^{2}\right)-c_{1} c_{2} s^{2}\right]}{c_{1}^{2} s^{3}} & -c_{2} & 0 \\
0 & -c_{2} & \frac{a_{\mathrm{c}}^{+}(s)\left(c_{2}^{2}+c_{3}^{2}\right)-c_{1} c_{2} s^{2}}{c_{3} s} & -c_{3} \\
0 & 0 & -c_{3} & \frac{c_{3} a_{\mathrm{c}}^{-}(s)}{s}
\end{array}\right]
$$

for any $s \in\left(\xi_{\mathrm{c}}, \tau_{\mathrm{c}}^{2}\right]$ are the only Jacobi matrices of the form $\mathrm{J}=\mathrm{J}\left(c_{2} \mathrm{c}_{z}(\omega), \omega\right)$ such that their Moore-Penrose inverses are $M$-matrices.

Proof. If $\mathrm{c}=\left(c_{1}, c_{2}, c_{3}\right) \in K$, then $\mathrm{c}=c_{2} \mathrm{c}(\omega)$ iff

$$
c_{1}=\frac{c_{2} \omega_{1} \omega_{3}}{\omega_{3}^{2}+\omega_{4}^{2}} \quad \text { and } \quad c_{3}=\frac{c_{2} \omega_{2} \omega_{4}}{\omega_{1}^{2}+\omega_{2}^{2}}
$$

that is, iff

$$
c_{1} \omega_{3}^{2}-c_{2} \omega_{1} \omega_{3}+c_{1} \omega_{4}^{2}=c_{3} \omega_{2}^{2}-c_{2} \omega_{2} \omega_{4}+c_{3} \omega_{1}^{2}=0 .
$$

Solving the above equations, we obtain that

$$
\omega_{2}=\frac{\omega_{4}}{2 c_{3}}\left(c_{2} \pm \sqrt{c_{2}^{2}-4 c_{3}^{2} \frac{\omega_{1}^{2}}{\omega_{4}^{2}}}\right) \text { and } \omega_{3}=\frac{\omega_{1}}{2 c_{1}}\left(c_{2} \pm \sqrt{c_{2}^{2}-4 c_{1}^{2} \frac{\omega_{4}^{2}}{\omega_{1}^{2}}}\right) .
$$

So, if we define $s=\frac{\omega_{4}}{\omega_{1}}$, necessarily $\tau_{\mathrm{c}}^{1}=\frac{2 c_{3}}{c_{2}} \leq s \leq \frac{c_{2}}{2 c_{1}}=\tau_{\mathrm{c}}^{2}$ and then we get that $\omega_{3}=a_{\mathrm{c}}^{ \pm}(s) \omega_{1}$ and $\omega_{2}=b_{\mathrm{c}}^{ \pm}(s) \omega_{1}$. Moreover, $\omega \in \Omega$ iff

$$
1=\omega_{1}^{2}\left[1+\left(b_{\mathrm{c}}^{ \pm}(s)\right)^{2}+\left(a_{\mathrm{c}}^{ \pm}(s)\right)^{2}+s^{2}\right]=\frac{\omega_{1}^{2} c_{2}}{c_{1} c_{3}}\left[c_{3} a_{\mathrm{c}}^{ \pm}(s)+c_{1} s b_{\mathrm{c}}^{ \pm}(s)\right] .
$$

In addition, $\omega \in \Omega_{0}$ iff $s=a_{\mathrm{c}}^{ \pm}(s) b_{\mathrm{c}}^{ \pm}(s)$ whereas $\omega \in \Omega_{+}$iff $s<a_{\mathrm{c}}^{ \pm}(s) b_{\mathrm{c}}^{ \pm}(s)$. Therefore, the result for matrices $\mathrm{J}_{c}^{j}(s), j=1,2,3$, follows from Proposition 2.1 applying Lemma 3.4 to the Identity (2.1). Analogous reasonings prove the claimed properties for the matrices $\mathrm{J}_{c}^{j}(s), j=4,5,6,7$.

REMARK 3.6. The strict monotonicity of $a_{\mathrm{c}}^{ \pm}$and $b_{\mathrm{c}}^{ \pm}$implies that $\mathrm{J}_{\mathrm{c}}^{j}(t)=\mathrm{J}_{\mathrm{c}}^{j}(s)$ iff $s=t$, for any $j=1, \ldots, 7$. Moreover, $\mathrm{J}_{\mathrm{c}}^{1}(t)=\mathrm{J}_{\mathrm{c}}^{2}(s)$ or $\mathrm{J}_{\mathrm{c}}^{4}(t)=\mathrm{J}_{\mathrm{c}}^{5}(s)$ iff $s=t=\tau_{\mathrm{c}}^{1}$, $\mathrm{J}_{\mathrm{c}}^{1}(t)=\mathrm{J}_{\mathrm{c}}^{3}(s)$ or $\mathrm{J}_{\mathrm{c}}^{6}(t)=\mathrm{J}_{\mathrm{c}}^{7}(s)$ iff $s=t=\tau_{\mathrm{c}}^{2}$. In the remaining cases, all matrices are pairwise different.

4. Jacobi matrices of the form $\mathrm{J}\left(t c_{y}(\omega), \omega\right)$. The last section in this work refers to the study of the Jacobi matrices $\mathrm{J}(\mathrm{c}, \omega)$, where $\mathrm{c}=c_{2} \mathrm{c}_{y}(\omega)$ and $\omega \in \Omega_{+}$. 
Recall that if $c=c_{2} c_{y}(\omega)$ and if we let $r^{2}=\omega_{1}^{2}+\omega_{2}^{2}, t^{2}=\omega_{2}^{2}+\omega_{3}^{2}$ and $s=\frac{\omega_{4}}{\omega_{1}}$, then

$$
c_{1}=\frac{c_{2} \omega_{1} \omega_{3} t^{2}}{\omega_{1}^{2} r^{2} s^{2}+\omega_{3}^{2}\left(1-r^{2}\right)} \quad \text { and } \quad c_{3}=\frac{c_{2} \omega_{1} \omega_{2} t^{2} s}{\omega_{1}^{2}\left(1-r^{2}\right)+\omega_{2}^{2} r^{2}}
$$

which, from Young's Inequality, implies that $4 c_{1} c_{3} r^{2}\left(1-r^{2}\right)<c_{2}^{2} t^{4}$ or, equivalently, that the conductance defined as $\mathrm{c}(r, t)=\left(c_{1} r \sqrt{1-r^{2}}, c_{2} t^{2}, c_{3} r \sqrt{1-r^{2}}\right)$, belongs to the cone $K$.

Next we prove that the variables $r, t$ and $s$ can be used to parametrize the set of weights.

LEMmA 4.1. Given $\omega \in \Omega$ there exist unique $r, t \in(0,1)$ and $s>0$ satisfying $s^{2}\left(r^{2}-t^{2}\right)<1-r^{2}<s^{2} r^{2}+t^{2}$ and such that $\omega=\omega(r, t, s)$ where

$$
\omega(r, t, s)=\sqrt{\frac{1-t^{2}}{1+s^{2}}}\left(1, \sqrt{\frac{s^{2} r^{2}+r^{2}+t^{2}-1}{1-t^{2}}}, \sqrt{\frac{s^{2}\left(t^{2}-r^{2}\right)+1-r^{2}}{1-t^{2}}}, s\right) .
$$

In addition, $\omega \in \Omega_{+}$iff one of the following properties hold:

1. $0<r<t \leq \sqrt{1-r^{2}}$ and $s>\sqrt{\frac{\left(1-r^{2}\right)\left(1-r^{2}-t^{2}\right)}{r^{2}\left(t^{2}-r^{2}\right)}}$.

2. $0<\sqrt{1-r^{2}}<t<r$ and $0<s<\sqrt{\frac{r^{2}\left(t^{2}-r^{2}\right)}{\left(1-r^{2}\right)\left(1-r^{2}-t^{2}\right)}}$.

3. $\sqrt{1-r^{2}}<t, r \leq t$ and $s>0$.

Proof. Clearly, $r^{2}=\omega_{1}^{2}(r, t, s)+\omega_{2}^{2}(r, t, s), 1-r^{2}=\omega_{3}^{2}(r, t, s)+\omega_{4}^{2}(r, t, s)$, which implies that $\omega(r, t, s) \in \Omega$ and moreover $t^{2}=\omega_{2}^{2}(r, t, s)+\omega_{3}^{2}(r, t, s)$.

On the other hand, $\omega(r, t, s)=\omega(\hat{r}, \hat{t}, \hat{s})$ iff $\hat{r}=r, \hat{t}=t$ and $\hat{s}=s$, so the uniqueness follows. On the other hand, if $\omega=\left(\omega_{1}, \omega_{2}, \omega_{3}, \omega_{4}\right)$ and consider the values $r^{2}=\omega_{1}^{2}+\omega_{2}^{2}, t^{2}=\omega_{2}^{2}+\omega_{3}^{2}$ and $s=\frac{\omega_{4}}{\omega_{1}}$ then

$$
\omega_{2}=\sqrt{r^{2}-\omega_{1}^{2}}, \quad \omega_{3}=\sqrt{t^{2}-r^{2}+\omega_{1}^{2}} \quad \text { and } \quad s^{2} \omega_{1}^{2}=1-t^{2}-\omega_{1}^{2},
$$

which implies that $\omega_{1}=\sqrt{\frac{1-t^{2}}{1+s^{2}}}$ and the first result follows. In addition, $\omega \in \Omega_{+}$iff

$$
0<\left(1+s^{2}\right)\left(s^{2} r^{2}\left(t^{2}-r^{2}\right)+\left(1-r^{2}\right)\left(r^{2}+t^{2}-1\right)\right)
$$

and the last claim follows. $\square$

In view of the above result, for any $r, t \in(0,1)$ we consider the interval $I_{r, t}$, defined as 
1. $I_{r, t}=\left(\sqrt{\frac{\left(1-r^{2}\right)\left(1-r^{2}-t^{2}\right)}{r^{2}\left(t^{2}-r^{2}\right)}},+\infty\right)$, if $0<r<t \leq \sqrt{1-r^{2}}$.

2. $I_{r, t}=\left(0, \sqrt{\frac{r^{2}\left(t^{2}-r^{2}\right)}{\left(1-r^{2}\right)\left(1-r^{2}-t^{2}\right)}}\right)$, if $0<\sqrt{1-r^{2}}<t<r$.

3. $I_{r, t}=(0,+\infty)$, if $\sqrt{1-r^{2}}<t$ and $r \leq t$.

4. $I_{r, t}=\emptyset$, otherwise.

Now, we obtain necessary and sufficient conditions on a conductance $\mathrm{c}$ and a weight $\omega \in \Omega_{+}$for the equality $c=c_{2} c_{y}(\omega)$ holds. First we observe that for any $r, t \in(0,1)$ we get that $\xi_{\mathrm{c}(r, t)}=\xi_{\mathrm{c}}=\sqrt{\frac{c_{3}}{c_{1}}}$.

Proposition 4.2. Given $\omega(r, t, s) \in \Omega_{+}$, then $\mathrm{c}=c_{2} \mathrm{c}_{y}(\omega(r, t, s))$ iff one of the following equalities holds:

1.- $s \in\left[\tau_{\mathrm{c}(r, t)}^{1}, \tau_{\mathrm{c}(r, t)}^{2}\right] \cap I_{r, t}$ and

$$
\frac{\sqrt{1-r^{2}}}{r} b_{\mathrm{c}(r, t)}^{+}(s)=\sqrt{\frac{s^{2} r^{2}+r^{2}+t^{2}-1}{1-t^{2}}}, \frac{r a_{\mathrm{c}(r, t)}^{+}(s)}{\sqrt{1-r^{2}}}=\sqrt{\frac{s^{2}\left(t^{2}-r^{2}\right)+1-r^{2}}{1-t^{2}}} .
$$

2.- $s \in\left[\tau_{c(r, t)}^{1}, \xi_{\mathrm{c}}\right) \cap I_{r, t}$ and

$$
\frac{\sqrt{1-r^{2}}}{r} b_{c(r, t)}^{-}(s)=\sqrt{\frac{s^{2} r^{2}+r^{2}+t^{2}-1}{1-t^{2}}}, \frac{r a_{\mathrm{c}(r, t)}^{+}(s)}{\sqrt{1-r^{2}}}=\sqrt{\frac{s^{2}\left(t^{2}-r^{2}\right)+1-r^{2}}{1-t^{2}}} .
$$

3.- $s \in\left(\xi_{\mathrm{c}}, \tau_{\mathrm{c}(r, t)}^{2}\right] \cap I_{r, t}$ and

$$
\frac{\sqrt{1-r^{2}}}{r} b_{\mathrm{c}(r, t)}^{+}(s)=\sqrt{\frac{s^{2} r^{2}+r^{2}+t^{2}-1}{1-t^{2}}}, \frac{r a_{\mathrm{c}(r, t)}^{-}(s)}{\sqrt{1-r^{2}}}=\sqrt{\frac{s^{2}\left(t^{2}-r^{2}\right)+1-r^{2}}{1-t^{2}}} .
$$

Proof. From Identities 4.1) we obtain

$$
\omega_{2}=\frac{\sqrt{1-r^{2}}}{r} b_{\mathrm{c}(r, t)}^{ \pm}(s) \omega_{1} \quad \text { and } \quad \omega_{3}=\frac{r}{\sqrt{1-r^{2}}} a_{\mathrm{c}(r, t)}^{ \pm}(s) \omega_{1} .
$$

Therefore, Lemma 3.4 implies that $\omega$ must satisfy one of the following identities:

1.- $\omega=k(s, r, t)\left(1, \frac{\sqrt{1-r^{2}}}{r} b_{\mathrm{c}(r, t)}^{+}(s), \frac{r}{\sqrt{1-r^{2}}} a_{\mathrm{c}(r, t)}^{+}(s), s\right)$, for $s \in\left[\tau_{\mathrm{c}(r, t)}^{1}, \tau_{\mathrm{c}(r, t)}^{2}\right]$;

2.- $\omega=k(s, r, t)\left(1, \frac{\sqrt{1-r^{2}}}{r} b_{\mathrm{c}(r, t)}^{-}(s), \frac{r}{\sqrt{1-r^{2}}} a_{\mathrm{c}(r, t)}^{+}(s), s\right)$, for $s \in\left[\tau_{\mathrm{c}(r, t)}^{1}, \xi_{\mathrm{c}}\right)$;

3.- $\omega=k(s, r, t)\left(1, \frac{\sqrt{1-r^{2}}}{r} b_{\mathrm{c}(r, t)}^{+}(s), \frac{r}{\sqrt{1-r^{2}}} a_{\mathrm{c}(r, t)}^{-}(s), s\right)$, for $s \in\left(\xi_{\mathrm{c}}, \tau_{\mathrm{c}(r, t)}^{2}\right]$, 
where $k(s, r, t)$ is a normalization factor so $\omega$ is unitary. The conclusions follow from the expression of the weight $\omega(r, t, s)$.

Remark 4.3. The above Proposition establishes necessary and sufficient conditions for the identity $\mathrm{c}=c_{2} c_{y}(\omega), \omega \in \Omega_{+}$, but given a conductance $\mathrm{c}$ it is very difficult to elucidate if there exist parameters $r, t$ and $s$ satisfying the equalities

$$
\begin{aligned}
& \frac{\sqrt{1-r^{2}}}{r} b_{\mathrm{c}(r, t)}^{ \pm}(s)=\sqrt{\frac{s^{2} r^{2}+r^{2}+t^{2}-1}{1-t^{2}}}, \\
& \frac{r}{\sqrt{1-r^{2}}} a_{\mathrm{c}(r, t)}^{ \pm}(s)=\sqrt{\frac{s^{2}\left(t^{2}-r^{2}\right)+1-r^{2}}{1-t^{2}}} .
\end{aligned}
$$

Therefore, we only consider some specific cases in which we can solve these non-linear equalities. Moreover, we mainly pay attention to those cases that are not covered in the preceding sections, that is, when the conductance does not belong to the cone $K$.

All cases where we tackle the system (4.2) involve the value $s=1$, that is equivalent to assume that $\omega_{1}=\omega_{4}$ and requires that $\tau_{c(r, t)}^{1} \leq 1 \leq \tau_{c(r, t)}^{2}$, or expressed in an equivalent form, that $t$ and $r$ must be chosen satisfying

$$
\max \left\{\frac{c_{1}}{c_{2}}, \frac{c_{3}}{c_{2}}\right\} \leq \frac{t^{2}}{2 r \sqrt{1-r^{2}}}
$$

Moreover, we will take into account that $\xi_{\mathrm{c}}$ is less than, equal to or greater than $1 \mathrm{iff}$ $c_{1}$ is less than, equal to or greater than $c_{3}$.

Assuming $s=1$, we also suppose that either $r=\sqrt{1-r^{2}}$; that is, $r=\frac{\sqrt{2}}{2}$, or $t=\sqrt{1-r^{2}}$, or $t=r$. In an equivalent way, we consider weights satisfying either $\omega_{1}=\omega_{4}, \omega_{2}=\omega_{3}$ and $\omega_{1}<\omega_{2}$, or $\omega_{1}=\omega_{3}=\omega_{4}$ and $\omega_{1}<\omega_{2}$ or $\omega_{1}=\omega_{2}=\omega_{4}$ and $\omega_{1}<\omega_{3}$, respectively. So, we consider the set of weights defined as

$$
\begin{aligned}
& \Omega_{1}=\left\{\omega \in \Omega: \omega_{1}=\omega_{4}, \omega_{2}=\omega_{3}, \omega_{1}<\omega_{2}\right\}, \\
& \Omega_{2}=\left\{\omega \in \Omega: \omega_{1}=\omega_{2}=\omega_{4}, \omega_{1}<\omega_{3}\right\}, \\
& \Omega_{3}=\left\{\omega \in \Omega: \omega_{1}=\omega_{3}=\omega_{4}, \omega_{1}<\omega_{2}\right\}
\end{aligned}
$$

and look for conductances of the form $c_{2} c_{y}(\omega)$ where $\omega \in \widehat{\Omega}=\Omega_{1} \cup \Omega_{2} \cup \Omega_{3}$. Observe that if $\omega \in \Omega_{1}$, then $c_{1}=c_{3}<c_{2} t^{2}$; if $\omega \in \Omega_{2}$, then $c_{3}=c_{2} t^{2}$ and when $\omega \in \Omega_{3}$, then $c_{1}=c_{2} t^{2}$

It is easy to check that in all the above cases, $s=1$ and either $r=\frac{\sqrt{2}}{2}$, or $t=\sqrt{1-r^{2}}$, or $t=r, I_{r, t}=(0,+\infty)$ and moreover, $\frac{\sqrt{2}}{2}<t<1$. In addition, in the 
three cases, System (4.2) is equivalent to

$$
\begin{aligned}
2 c_{3}^{2} r^{2}\left[\left(r^{2}+t^{2}-1\right)\left(2 r^{2}-1\right)+r^{2}\right] & =c_{2}^{2} t^{4}\left(1-t^{2}\right) \\
& \pm c_{2} t^{2}\left(1-t^{2}\right) \sqrt{c_{2}^{2} t^{4}-4 c_{3}^{2} r^{2}\left(1-r^{2}\right),} \\
2 c_{1}^{2}\left(1-r^{2}\right)\left[\left(r^{2}-t^{2}\right)\left(2 r^{2}-1\right)+1-r^{2}\right] & =c_{2}^{2} t^{4}\left(1-t^{2}\right) \\
& \pm c_{2} t^{2}\left(1-t^{2}\right) \sqrt{c_{2}^{2} t^{4}-4 c_{1}^{2} r^{2}\left(1-r^{2}\right)}
\end{aligned}
$$

To solve the above system it will be useful to consider $f, g:\left(\frac{\sqrt{2}}{2}, 1\right) \longrightarrow \mathbb{R}$, defined as $f(t)=2 t^{3} \sqrt{1-t^{2}}$ and $g(t)=\frac{4 t^{4} \sqrt{\left(1-t^{2}\right)\left(3 t^{2}-1\right)}}{\left(2 t^{2}-1\right)^{2}+t^{2}}$, respectively; see Figure 4.1
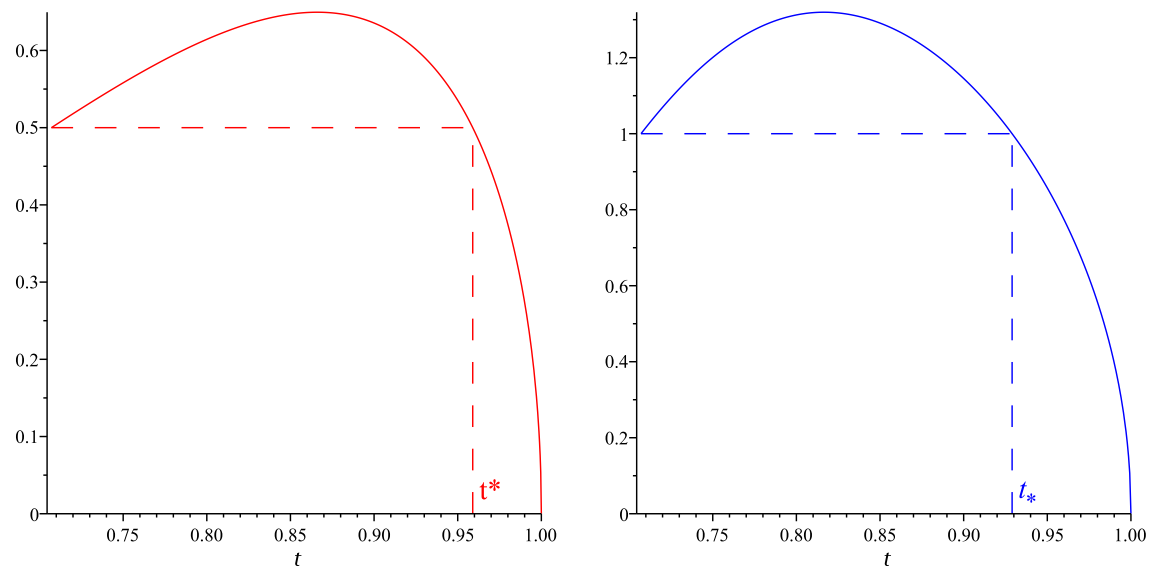

FIG. 4.1. Graphs of functions $f$ (left) and $g$ (right)

Lemma 4.4. The following properties hold:

1. $f(t) \leq f\left(\frac{\sqrt{3}}{2}\right)=\frac{3 \sqrt{3}}{8}, g(t) \leq g\left(\frac{\sqrt{6}}{3}\right)=\frac{16 \sqrt{3}}{21}$ and $g(t)<4 t^{4}$ for $\frac{\sqrt{2}}{2}<t<1$.

2. $f(t)=\frac{1}{2}$ iff $t=t^{*}=\frac{\sqrt{6}}{6} \sqrt{\sqrt[3]{19+3 \sqrt{33}}+\frac{4}{\sqrt[3]{19+3 \sqrt{33}}}+1} \simeq 0.9589803845$

and moreover $f(t)>\frac{1}{2}$ iff $\frac{\sqrt{2}}{2}<t<t^{*}$.

3. $g(t)=1$ iff $t=t_{*} \simeq 0.9289644667$ and moreover, $g(t)>1$ iff $\frac{\sqrt{2}}{2}<t<t_{*}$

If we consider the 3-dimensional cone, see Figure 4.2 .

$$
K_{y}^{1}=\left\{\left(c, c_{2}, c\right) \in(0,+\infty)^{3}: c_{2} \geq \frac{8 \sqrt{3}}{9} c\right\}
$$


then, the cone of conductances in $K_{y}^{1}$ not considered in the previous sections is

$$
K_{y}^{1} \backslash K=\left\{\left(c, c_{2}, c\right) \in(0,+\infty)^{3}: \frac{8 \sqrt{3}}{9} c \leq c_{2}<2 c\right\} .
$$
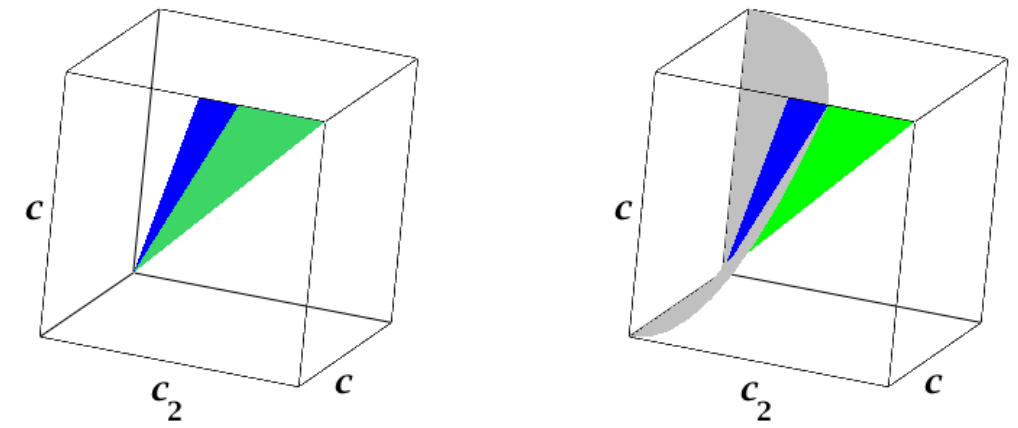

FIG. 4.2. Cone $K_{y}^{1}$ of feasible conductances. $K_{y}^{1} \cap K$ (green) and $K_{y}^{1} \backslash K$ (blue)

Proposition 4.5. Given $\mathrm{c}=\left(c, c_{2}, c\right) \in K_{y}^{1}$, then all Jacobi matrices of the form $\mathrm{J}\left(c_{2} c_{y}(\omega), \omega\right), \omega \in \widehat{\Omega}$, whose Moore-Penrose inverse is an $M$-matrix are given by

$$
\mathrm{J}_{\mathrm{c}}^{8}(t)=\left[\begin{array}{cccc}
2 t^{3} c_{2} & -c & 0 & 0 \\
-c & c_{2}\left(1+2 t-2 t^{3}\right) & -c_{2} & 0 \\
0 & -c_{2} & c_{2}\left(1+2 t-2 t^{3}\right) & -c \\
0 & 0 & -c & 2 t^{3} c_{2}
\end{array}\right]
$$

where $\frac{\sqrt{2}}{2}<t<1$ satisfies $c=c_{2} f(t)$.

Proof. We can only consider the case ++ in System (4.3), since $1=\xi_{\mathrm{c}}$. Therefore, when either $t=r$ or $t=\sqrt{1-r^{2}}$, then System (4.3) does not have any solution, since then it is equivalent to $c=c_{2} t^{2}=\frac{g(t)}{4 t^{2}} c_{2}$, which would imply that $g(t)=4 t^{4}$ and this equation does not have solutions on $\left(\frac{\sqrt{2}}{2}, 1\right)$.

When $r=\frac{\sqrt{2}}{2}$, then $c<c_{2} t^{2}$ and the two equations of System (4.3) are equal and become

$$
c^{2}-2 c_{2}^{2} t^{4}\left(1-t^{2}\right)=2 c_{2} t^{2}\left(1-t^{2}\right) \sqrt{c_{2}^{2} t^{4}-c^{2}}
$$


which implies that $c=c_{2} f(t)$ and hence, $\omega\left(\frac{\sqrt{2}}{2}, t, 1\right)=\frac{\sqrt{2}}{2}\left(\sqrt{1-t^{2}}, t, t, \sqrt{1-t^{2}}\right)$. The result follows taking into account Identity (2.1).

REMARK 4.6. When $c_{2} \geq \frac{8 \sqrt{3}}{9} c$, there always exits $t \in\left(\frac{\sqrt{2}}{2}, 1\right)$ satisfying $c=$ $f(t) c_{2}$. Moreover, $\mathrm{J}_{\mathrm{c}}^{8}\left(t^{*}\right)$ is a Jacobi matrix associated with the conductance $(c, 2 c, c)$, other than the combinatorial Laplacian, see Remark 3.2, such that its Moore-Penrose inverse is also an $M$-matrix. In addition, when $\mathrm{c} \in \bar{K}$, there exists a unique $t \in\left[t^{*}, 1\right)$ such that $c=f(t) c_{2}$, whereas when $\frac{8 \sqrt{3}}{9} c<c_{2}<2 c$, there exist only exactly two values $\frac{\sqrt{2}}{2}<t_{1}<\frac{\sqrt{3}}{2}<t_{2}<t^{*}$ such that $c=f\left(t_{1}\right) c_{2}=f\left(t_{2}\right) c_{2}$. When $c \in K$, these matrices are different from all matrices in Theorem 3.5

If we consider now the 3-dimensional cone, see Figure 4.3

$$
\begin{aligned}
K_{y}^{2} & =\left\{\left(c_{1}, c_{2}, c_{3}\right) \in(0,+\infty)^{3}: c_{1} \neq c_{3}, c_{1}<c_{2}<2 c_{1}, 4 c_{1} c_{3}=c_{2}^{2} g\left(\sqrt{\frac{c_{1}}{c_{2}}}\right)\right\} \\
& \cup\left\{\left(c_{1}, c_{2}, c_{3}\right) \in(0,+\infty)^{3}: c_{1} \neq c_{3}, c_{3}<c_{2}<2 c_{3}, \quad 4 c_{1} c_{3}=c_{2}^{2} g\left(\sqrt{\frac{c_{3}}{c_{2}}}\right)\right\},
\end{aligned}
$$

then, the cone of conductances in $K_{y}^{2}$ not considered in the previous sections is

$$
\begin{aligned}
K_{y}^{2} \backslash K & =\left\{\left(c_{1}, c_{2}, c_{3}\right) \in(0,+\infty)^{3}: c_{1} \neq c_{3}, \frac{c_{1}}{t_{*}^{2}} \leq c_{2}<2 c_{1}, 4 c_{1} c_{3}=c_{2}^{2} g\left(\sqrt{\frac{c_{1}}{c_{2}}}\right)\right\} \\
& \cup\left\{\left(c_{1}, c_{2}, c_{3}\right) \in(0,+\infty)^{3}: c_{1} \neq c_{3}, \frac{c_{3}}{t_{*}^{2}} \leq c_{2}<2 c_{3}, 4 c_{1} c_{3}=c_{2}^{2} g\left(\sqrt{\frac{c_{3}}{c_{2}}}\right)\right\} .
\end{aligned}
$$
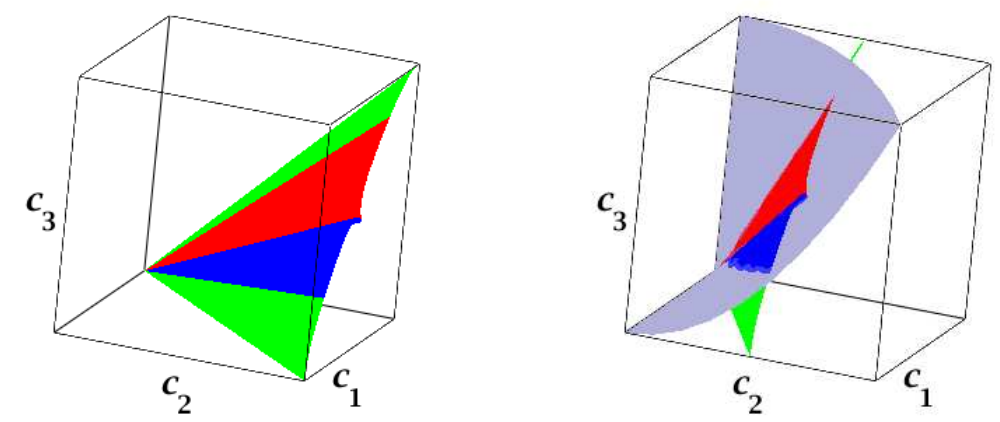

Fig. 4.3. Cone $K_{y}^{2}: K_{y}^{2} \cap K$ (green), $K_{y}^{2} \backslash K c_{1}>c_{3}$ (blue) and $K_{y}^{2} \backslash K c_{1}<c_{3}$ (red)

Observe that if $\left(c_{1}, c_{2}, c_{3}\right) \in K_{y}^{2}$ then $c_{1} \neq c_{3}$, otherwise if $t=\sqrt{\frac{c_{1}}{c_{2}}}$, then $4 t^{4}=g(t)$ 
which is impossible.

Proposition 4.7. Given $\mathrm{c}=\left(c_{1}, c_{2}, c_{3}\right) \in K_{y}^{2}$, then the only Jacobi matrix of the form $\mathrm{J}\left(c_{2} c_{y}(\omega), \omega\right), \omega \in \widehat{\Omega}$, whose Moore-Penrose inverse is an $M$-matrix is

$$
\mathrm{J}_{\mathrm{c}}^{9}=\left[\begin{array}{cccc}
c_{1} \sqrt{\frac{3 c_{1}-c_{2}}{c_{2}-c_{1}}} & -c_{1} & 0 & 0 \\
-c_{1} & \left(c_{1}+c_{2}\right) \sqrt{\frac{c_{2}-c_{1}}{3 c_{1}-c_{2}}} & -c_{2} & 0 \\
0 & -c_{2} & c_{3}+c_{2} \sqrt{\frac{3 c_{1}-c_{2}}{c_{2}-c_{1}}} & -c_{3} \\
0 & 0 & -c_{3} & c_{3}
\end{array}\right]
$$

when $c_{3}<c_{1}$, or

$$
\mathrm{J}_{\mathrm{c}}^{10}=\left[\begin{array}{cccc}
c_{1} & -c_{1} & 0 & 0 \\
-c_{1} & c_{1}+c_{2} \sqrt{\frac{3 c_{3}-c_{2}}{c_{2}-c_{3}}} & -c_{2} & 0 \\
0 & -c_{2} & \left(c_{2}+c_{3}\right) \sqrt{\frac{c_{2}-c_{3}}{3 c_{3}-c_{2}}} & -c_{3} \\
0 & 0 & -c_{3} & c_{3} \sqrt{\frac{3 c_{3}-c_{2}}{c_{2}-c_{3}}}
\end{array}\right]
$$

when $c_{1}<c_{3}$.

Proof. If $c_{3}<c_{1}$, then $\xi_{\mathrm{c}}<1$ and hence only the case +- in System (4.3) makes sense. Moreover, the second equation implies that $c_{1}=c_{2} t^{2}$, and hence $c_{1}<c_{2}<2 c_{1}$, whereas the first equation is

$$
c_{2}\left(1-t^{2}\right) \sqrt{c_{2}^{2} t^{4}-4 c_{3}^{2} t^{2}\left(1-t^{2}\right)}=2 c_{3}^{2}\left[\left(2 t^{2}-1\right)^{2}+t^{2}\right]-c_{2}^{2} t^{2}\left(1-t^{2}\right),
$$

which implies that $c_{3}=\frac{c_{2} g(t)}{4 t^{2}}=\frac{c_{2}^{2}}{4 c_{1}} g\left(\sqrt{\frac{c_{1}}{c_{2}}}\right)$, since $t=\sqrt{\frac{c_{1}}{c_{2}}}$ and hence,

$$
\omega(t, t, 1)=\sqrt{\frac{1-t^{2}}{2}}\left(1, \sqrt{\frac{3 t^{2}-1}{1-t^{2}}}, 1,1\right)=\sqrt{\frac{c_{2}-c_{1}}{2 c_{2}}}\left(1, \sqrt{\frac{3 c_{1}-c_{2}}{c_{2}-c_{1}}}, 1,1\right) .
$$

On the other hand, if $c_{1}<c_{3}$, then $1<\xi_{\mathrm{c}}$ and hence only the case -+ in System (4.3) makes sense. Now, the first equation implies that $c_{3}=c_{2} t^{2}$, and hence $c_{3}<c_{2}<2 c_{3}$, whereas the second equation is

$$
c_{2}\left(1-t^{2}\right) \sqrt{c_{2}^{2} t^{4}-4 c_{1}^{2} t^{2}\left(1-t^{2}\right)}=2 c_{1}^{2}\left[\left(2 t^{2}-1\right)^{2}+t^{2}\right]-c_{2}^{2} t^{2}\left(1-t^{2}\right),
$$

which implies that $c_{1}=\frac{c_{2} g(t)}{4 t^{2}}=\frac{c_{2}^{2}}{4 c_{1}} g\left(\sqrt{\frac{c_{3}}{c_{2}}}\right)$, since $t=\sqrt{\frac{c_{3}}{c_{2}}}$ and hence,

$$
\omega\left(\sqrt{1-t^{2}}, t, 1\right)=\sqrt{\frac{1-t^{2}}{2}}\left(1,1, \sqrt{\frac{3 t^{2}-1}{1-t^{2}}}, 1\right)=\sqrt{\frac{c_{2}-c_{3}}{2 c_{2}}}\left(1,1, \sqrt{\frac{3 c_{3}-c_{2}}{c_{2}-c_{3}}}, 1\right) .
$$


The results follow taking into account Identity (2.1).

REMARK 4.8. When $\mathrm{c} \in \partial K$; that is when $c_{2}=2 \sqrt{c_{1} c_{3}}$, then the matrix $\mathrm{J}_{\mathrm{c}}^{9}$ if $c_{3}<c_{1}$ or $\mathrm{J}_{c}^{10}$ if $c_{1}<c_{3}$, is different from $\mathrm{J}_{\mathrm{c}}$, the matrix obtained in Theorem 3.1. When $\mathrm{c} \in K$, matrices $\mathrm{J}_{\mathrm{c}}^{8}$ and $\mathrm{J}_{\mathrm{c}}^{9}$ do not coincide and they are different from all matrices in Theorem 3.5.

\section{REFERENCES}

[1] R.B. Bapat, S.J. Kirkland, S. Pati. The perturbed Laplacian matrix of a graph. Linear Multinear Algebra, 49:219-242, 2001.

[2] E. Bendito, A. Carmona, A.M. Encinas and M. Mitjana. Generalized inverses of symmetric M-matrices. Linear Algebra Appl., 432:2438-2454, (2010).

[3] E. Bendito, A. Carmona, A.M. Encinas and M. Mitjana. The $M$-matrix inverse problem for singular and symmetric Jacobi matrices. Linear Algebra Appl., 436:1090-1098 (2012).

[4] Y. Chen, S. J. Kirkland and M. Neumann. Group generalized inverses of $M$-matrices associated with periodic and nonperiodic Jacobi matrices. Linear Multilinear Algebra, 39:325-340, (1995).

[5] Y. Chen and M. Neumann. $M$-matrix generalized inverses of $M$-matrices. Linear Algebra Appl., 256:263-285, (1997). 